\title{
Knockout of krüppel-like factor 10 suppresses hepatic cell proliferation in a partially hepatectomized mouse model
}

\author{
SEUNG-HO HEO ${ }^{1,2}$, EUI-SUK JEONG ${ }^{1,3}$, KYOUNG-SUN LEE ${ }^{1,4}$, \\ JIN-HEE SEO ${ }^{1,5}$, WOON-KYU LEE ${ }^{6}$ and YANG-KYU CHOI ${ }^{1}$ \\ ${ }^{1}$ Department of Laboratory Animal Medicine, College of Veterinary Medicine, \\ Konkuk University, Seoul 05029; ${ }^{2}$ Asan Institute for Life Sciences, University of Ulsan College of Medicine, \\ Seoul 05505; ${ }^{3}$ Laboratory Animal Center, Daegu-Gyeongbuk Medical Innovation Foundation, \\ Daegu 41061; ${ }^{4}$ Laboratory Animal Center, Osong Medical Innovation Foundation, Chungbuk 28160; \\ ${ }^{5}$ Laboratory Animal Facility, Korea Institute of Radiological and Medical Sciences, Seoul 01812; ${ }^{6}$ Laboratory of \\ Developmental Genetics, College of Medicine, Inha University, Incheon 22212, Republic of Korea
}

Received February 18, 2016; Accepted January 26, 2017

DOI: $10.3892 / \mathrm{ol} .2017 .6044$

\begin{abstract}
The liver has marked regenerative capabilities, and numerous signaling pathways are involved in liver regeneration. The transforming growth factor- $\beta$ (TGF- $\beta$ )/Smad pathway, which is also involved in liver regeneration, regulates numerous biological processes. Krüppel-like factor 10 (KLF10) has been reported to activate the TGF- $\beta / \mathrm{Smad}$ signaling pathway; however, the exact functions of KLF10 under various pathophysiological conditions remain unclear. In the present study, the role of KLF10 in liver regeneration following partial hepatectomy $(\mathrm{PH})$ was investigated using KLF10-knockout (KO) mice. KLF10-KO mice exhibited lower liver/body weight ratios and 5-bromo-2-deoxy-uridine labeling indices compared with wild-type (WT) mice, and significant differences $(\mathrm{P}=0.028)$ were obtained at $72 \mathrm{~h}$ after $\mathrm{PH}$. To understand the causes of the gross and histopathological findings, the expression levels of the components of the TGF- $\beta /$ Smad pathway were examined using reverse transcription-quantitative polymerase chain reaction and western blot analysis. The mRNA and protein levels of Smad3, p15, TGF- $\beta 1$ and TGF- $\beta$ receptor 1 were significantly increased, while those of cMyc and cyclin D1 (proliferation-associated genes) were significantly lower in the liver tissues of the KLF10-KO mice compared with those of the WT mice at $72 \mathrm{~h}$ post-PH. These results indicated that KLF10-KO may exhibit
\end{abstract}

Correspondence to: Dr Yang-Kyu Choi, Department of Laboratory Animal Medicine, College of Veterinary Medicine, Konkuk University, 120 Neungdong-Ro, Gwangjin, Seoul 05029, Republic of Korea

E-mail: yangkyuc@konkuk.ac.kr

Key words: krüppel-like factor 10, liver regeneration, partial hepatectomy, proliferation, transforming growth factor- $\beta / \mathrm{Smad}$ pathway antiproliferative effects on liver regeneration following $\mathrm{PH}$, through strengthening the TGF- $\beta /$ Smad signaling pathway in a delayed manner.

\section{Introduction}

The liver has excellent regenerative capacities. Liver cells are quiescent under normal conditions; however, they enter the cell cycle when damaged and proliferate until the original liver volume is restored (1). A number of conditions may alter liver mass, including surgical resection, chemicals and pathogens (2-4). In the situation of liver cancer or hepatocirrhosis, surgical resection is a standard medical therapy. Cirrhosis of the liver is one of the leading causes of mortality, and liver cancer is the third most common cancer and the second leading cause of cancer-associated mortality worldwide $(5,6)$.

Numerous signaling pathways are involved in liver regeneration, including pathways involving hepatocyte growth factor, epidermal growth factor, interleukin 6, tumor necrosis factor- $\alpha$ and transforming growth factor (TGF)- $\alpha$ and $\beta(2,7)$. Among these, the TGF- $\beta /$ Smad pathway is reported to suppress cellular proliferation and to regulate numerous biological processes; however, the responses to TGF- $\beta$ differ according to cell or tissue type and the microenvironment (8-10).

Activation of secreted TGF- $\beta$ and assembly of TGF- $\beta$ receptor type 1 and 2 (TGF- $\beta$ R 1 and 2 ) in the cellular membrane is the first step in the TGF- $\beta /$ Smad pathway. Subsequently, TGF- $\beta$ R 2 phosphorylates and activates TGF- $\beta$ $\mathrm{R} 1$, which in turn phosphorylates cytoplasmic Smad2 and Smad3 [also termed receptor-Smads (R-Smads)]. Activated $\mathrm{R}$-Smads bind Smad4 and move into the nucleus, and the Smads complex, along with co-factors, positively or negatively regulates the expression of target genes (11-14). In cellular proliferation, cMyc and cyclin D1 genes or proteins are downregulated, while the expression of p15, p21 and Smad2 genes is upregulated following activation of the TGF- $\beta / \mathrm{Smad}$ pathway. In non-Smad pathways, the TGF- $\beta$ receptor activates 
other proteins, including RAS, phosphoinositide 3-kinase and FAS (12,14-16).

As a target gene of TGF- $\beta$, KLF10 may regulate the TGF- $\beta /$ Smad pathway. KLF10 may enhance Smad2, p21 and plasminogen activator inhibitor-1 expression, and repress the transcription of the Smad7 gene. KLF10 also plays important roles in numerous biological processes, and it has been reported to inhibit proliferation and induce apoptosis in several cell types $(10,17,18)$. However, the role of KLF10 in various pathophysiological conditions remains unclear.

Partial hepatectomy (PH), resulting in the removal of $~ 70 \%$ of the liver, is widely utilized for studies of liver regeneration, acute liver failure and the metastasis of liver cancer $(19,20)$. KLF10 is known as a potential antiproliferative gene; however, to the best of our knowledge, there are no reports on the role of KLF10 in liver regeneration $(10,21)$. In the present study, to elucidate the role of the KLF10 gene in liver regeneration following tissue loss, molecular and histopathological analyses were conducted using KLF10-knockout (KO) mice following a $\mathrm{PH}$ that removed two-thirds of the liver.

\section{Materials and methods}

Animals. All procedures were approved by the Institutional Animal Care and Use Committee of Konkuk University (Seoul, South Korea). Three pairs of 8-week-old KLF10-KO C57BL/6 J mice (age: 54-57 days, average 8 weeks; body weight: $23.1-24.9 \mathrm{~g}$, average $24.1 \mathrm{~g}$ ) were kindly provided by Professor Woon-Kyu Lee (Inha University, Incheon, Korea) (22). and five pairs of 6-week-old C57BL/6J mice (age: 32-35 days old, average 6 weeks old; body weight: 21.9-25.1 g, average $23.7 \mathrm{~g}$ ) were obtained from the Korea Research Institute of Bioscience and Biotechnology (Daejeon, Korea). All mice were bred in the laboratory animal breeding room under specific pathogen-free conditions to produce the KO and wild-type (WT) mice groups. For genotyping each mouse, DNA samples were isolated from all mice tails using the Genomic DNA extraction kit (Bioneer Corporation, Daejeon, Korea) and subjected to polymerase chain reaction (PCR) using the AccuPower ${ }^{\circledR}$ PCR PreMix (\#K-2016; Bioneer Corporation). The DNA primers for genotyping were: KLF10 forward, CCTTCCTGCCAACAACTCTC and reverse, TCT GAGGAGTGACCCTTGCT; and KLF10-KO forward, TCG CCTTCTTGACGAGTTCT (12) and reverse, TCTGAGGAGTGACCCTTGCT. The cycling conditions were initial denaturation for $5 \mathrm{~min}$ at $95^{\circ} \mathrm{C}$, followed by 30 cycles of $1 \mathrm{~min}$ at $95^{\circ} \mathrm{C}, 1 \mathrm{~min}$ at annealing temperature, $1 \mathrm{~min}$ at $72^{\circ} \mathrm{C}$ and an additional $10 \mathrm{~min}$ at $72^{\circ} \mathrm{C}$ for final elongation. After the reaction, the PCR samples were electrophoresed on a $1.5 \%$ agarose gel. The size of KLF10 KO gene is 658 base pairs (bp) and that of WT gene is $248 \mathrm{bp}$.

Experimental design and surgical procedure. At 8 weeks of age, a two-thirds $\mathrm{PH}$ was performed. Subsequent to anaesthetizing with Zoletil (Virbac Corporation, Fort Worth, TX, USA) and Rompun (Bayer Korea, Ltd., Seoul, Korea), the middle abdominal skin and linea alba were incised. The left lateral and median liver lobes were excised and the peritoneum and the skin were closed (20). From each group of KLF10-KO and WT mice, 3-5 animals were sacrificed at $0,24,48$ or $72 \mathrm{~h}$
post-PH using the $\mathrm{CO}_{2}$ euthanasia chamber $(\mathrm{n}=3, \mathrm{n}=5, \mathrm{n}=5$ and $\mathrm{n}=5$, respectively). At $2 \mathrm{~h}$ prior to euthanasia, $100 \mathrm{mg} / \mathrm{kg}$ 5-bromo-2-deoxy-uridine (BrdU; Sigma-Aldrich; EMD Millipore, Billerica, MA, USA) was injected intraperitoneally. At the time of sacrifice, all mice were grossly examined and blood was collected from the caudal vena cava. The liver was excised and weighed. The upper right lateral lobe was fixed in $10 \%$ neutral-buffered formalin for histopathological analysis and the other hepatic tissues were frozen for subsequent analyses.

Histological and immunohistochemical analysis. Fixed tissues were processed routinely for paraffin sectioning. Liver tissues were then embedded in paraffin and cut into $4-\mu \mathrm{m}$ thick sections. The sections were deparaffinized, rehydrated and stained with hematoxylin and eosin (H\&E) (Sigma-Aldrich; EMD Millipore). For immunohistochemistry, serial sections were cut, deparaffinized, rehydrated and serial incubated in $1.0 \% \mathrm{H}_{2} \mathrm{O}_{2}, 2 \mathrm{~N} \mathrm{HCl}$ and $0.1 \%$ trypsin. The slides were pre-incubated with normal blocking serum (Vectastain ABC kit; \#PK-6102; Vector Laboratories, Inc., Burlingame, CA, USA) according to the manufacturer's protocol and then incubated with anti-BrdU antibody (\#B8434; dilution, 1:500; Sigma-Aldrich) for $2 \mathrm{~h}$ at room temperature. Detection of the BrdU was performed using biotinylated secondary antibodies (Vectastain ABC kit; \#PK-6102; dilution, 1:200; Vector Laboratories, Inc.; ), avidin-coupled peroxidase (Vectastain $A B C$ kit; Vector Laboratories, Inc.), and diaminobenzidine (DAB; DAB substrate kit; Vector Laboratories, Inc.).

Reverse transcription-quantitative polymerase chain reaction $(R T-q P C R)$. Total RNA was prepared from frozen liver tissues using TRIzol (Invitrogen; Thermo Fisher Scientific, Inc., Waltham, MA, USA) and was reverse transcribed into cDNA using moloney-murine leukemia virus reverse transcriptase (Invitrogen; Thermo Fisher Scientific, Inc.). The cDNA was used as a template for amplification in the PCR using the AccuPower ${ }^{\circledR}$ PCR PreMix (\#K-2016; Bioneer Corporation). Smad2, Smad3, Smad4, Smad7, p15, p21, TGF- $\beta$ R1, TGF- $\beta$ $\mathrm{R} 2$, cMyc, cyclin D1 and $\beta$-actin mRNA expression level were analyzed using the RT-qPCR method, as described by Shiao (23), with the previously described primers (12). The cycling conditions were initial denaturation for $5 \mathrm{~min}$ at $95^{\circ} \mathrm{C}$, followed by 35 cycles of $1 \mathrm{~min}$ at $95^{\circ} \mathrm{C}, 1 \mathrm{~min}$ at annealing temperature, $1 \mathrm{~min}$ at $72^{\circ} \mathrm{C}$ and an additional $10 \mathrm{~min}$ at $72^{\circ} \mathrm{C}$ for final elongation. After the reaction, the PCR mixtures were electrophoresed on a $1.5 \%$ agarose gel. Band intensities were quantified using ImageQuant Software (Image Lab V4.0; Bio-Rad Laboratories, Inc., Hercules, CA, USA) and were normalized to the transcription levels of $\beta$-actin. Each sample was tested in triplicate.

Western blot analysis. Protein was extracted from the liver using the extraction solution (Pro-Prep ${ }^{\mathrm{TM}}$; Intron Biotechnology, Inc., Seongnam, Korea). The protein concentrations were determined using the bicinchoninic kit (Pierce; Thermo Fisher Scientific, Inc.). Subsequent to being transferred to nitrocellulose membranes, the proteins were blocked with $5 \%$ skimmed milk and then incubated overnight with specific antibodies against $\beta$-actin (\#sc-47778; dilution, 1:500), Smad4 (\#sc-7966; dilution, 1:500), Smad7 (\#sc-11392; dilution, 
1:1,000), p15 (\#sc-65223; dilution, 1:200), p21 (\#sc-817; dilution, 1:500), TGF- $\beta$ R1 (\#sc-398; dilution, 1:200), cMyc (\#sc-56505; dilution, 1:200) (Santa Cruz Biotechnology, Inc., Dallas, TX, USA), Smad2 (\#3122; dilution, 1:1,000), Smad3 (\#9513; dilution, 1:200), p27 (\#2552; dilution, 1:500), TGF- $\beta$ R2 (\#11888, dilution, 1:500) and cyclin D1 (\#2922; dilution, 1:500; all from Cell Signaling Technology, Inc., Danvers, MA, USA) at $4^{\circ} \mathrm{C}$. Subsequently, the membranes were washed with TBS with Tween-20 and incubated for $1 \mathrm{~h}$ with either horseradish peroxidase-conjugated anti-rabbit (\#sc-2054; dilution, 1:1,000) or anti-mouse secondary antibodies (\#sc-2031; dilution, 1:1,000; Santa Cruz Biotechnology, Inc.) at room temperature. Specific antibodies were detected with an electrochemiluminescence test kit (KPL, Inc., Gaithersburg, MD, USA). The band intensities were quantified using Image Lab V4.0 (Bio-Rad Laboratories, Inc.) and were normalized to $\beta$-actin expression (12).

Statistical analysis. Statistical analysis was performed using SPSS V14.0 software (SPSS, Inc., Chicago, IL, USA). Statistically significant differences between the studied groups were evaluated using Student's unpaired t-test. $\mathrm{P}<0.05$ was considered to indicate a statistically significant difference.

\section{Results}

Gross findings. At 0, 24, 48 and $72 \mathrm{~h}$ post-PH, the body and liver weights of all mice were measured. Macroscopically, the volume of the remaining liver tissues increased from $48 \mathrm{~h}$ post-PH. The color of the liver subsequent to $\mathrm{PH}$ was paler than that of normal liver tissues from $48 \mathrm{~h}$ post- $\mathrm{PH}$. At the time of sacrifice, the liver was removed and weighed to examine the liver/body weight ratio. The mean of the ratios at $0,24,48$ and $72 \mathrm{~h}$ post-PH for the WT mice vs. KLF10-KO mice was 0.0194 vs. $0.0180,0.0210$ vs. 0.0194 , 0.0275 vs. 0.0257 and 0.0361 vs. 0.0307 , respectively (Fig. 1). Overall, KLF10-KO mice exhibited lower ratios compared with WT mice, and a significant difference was observed at 72 h post- $\mathrm{PH}(\mathrm{P}=0.028)$.

Histopathological analysis and BrdU staining. H\&E staining revealed no marked features at 0 and $24 \mathrm{~h}$ post-PH. Pale cytoplasm and hypertrophy of hepatocytes was observed from $48 \mathrm{~h}$ post-PH (Fig. 2A). Mitotic figures, mainly in the centrilobular area, were the most frequent at $48 \mathrm{~h}$ post-PH, and these remained numerous at $72 \mathrm{~h}$ post-PH. Until $24 \mathrm{~h}$ post-PH, few mitotic figures were observed (Fig. 2A). Similarly, upon BrdU staining to analyze the proliferative potential of cells, the proportion of labeled nuclei was markedly increased at $48 \mathrm{~h}$ post-PH (Fig. 2B). The number of BrdU-positive nuclei was counted in an area of $0.1 \mathrm{~mm}^{2}$ under a light microscope at x400 magnification. While calculating the BrdU labeling indices, the number of mitotic figures was added due to coarse staining of nuclei that were in the division stage (Fig. 2B). The mean of the BrdU labeling indices at 0, 24, 48 and $72 \mathrm{~h}$ post-PH for WT mice vs. KLF10-KO mice was 0.60 vs. 0.87 , 1.12 vs. $1.30,72.85$ vs. 59.45 and 40.1 vs. 26.56, respectively (Fig. 2C). Overall, KLF10-KO mice exhibited lower indices compared with WT mice from $48 \mathrm{~h}$ post- $\mathrm{PH}$, and a significant difference was observed at $72 \mathrm{~h}$ post-PH $(\mathrm{P}=0.036)$.

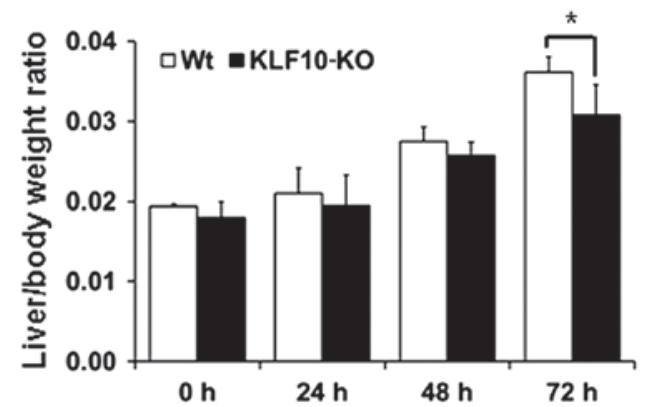

Figure 1. Liver/body weight ratio following PH. KLF10-KO mice exhibited lower ratios compared with WT mice, and a significant difference was observed at $72 \mathrm{~h}$ post-PH. ${ }^{*} \mathrm{P}<0.05$ by unpaired Student's t-test. KLF10, krüppel-like factor 10; KO, knockout; WT, wild-type; $\mathrm{PH}$, partial hepatectomy.

Expression of TGF- $\beta /$ Smad pathway genes. To determine the causes for the gross and histological findings, the expression of the components of the TGF- $\beta$ /Smads signaling pathway, which is known to be regulated by KLF10 (18), was analyzed. Smad2, Smad7 and p21, which have previously been reported to be target genes of KLF10 (10,17), and Smad3, Smad4, TGF- $\beta 1$, TGF- $\beta$ R1, TGF- $\beta$ R2, p15, p21, cMyc and cyclin D1, which are mediators or target genes of the TGF- $\beta /$ Smad pathway $(14,15)$, were analyzed by RT-qPCR. Smad2, one of the R-Smads, exhibited significantly lower transcription levels in KLF10-KO mice compared with WT mice from $24 \mathrm{~h}$ post-PH $(24 \mathrm{~h}$, $\mathrm{P}=0.034 ; 48$ h, $\mathrm{P}=0.036 ; 72$ h, $\mathrm{P}=0.028$; Fig. 3A). However, mRNA levels of Smad3 (another R-Smad) were increased in KLF10-KO mice compared with WT mice at all time points, and significant differences were observed from $48 \mathrm{~h}$ post-PH (48 h, $\mathrm{P}=0.039 ; 72$ h, $\mathrm{P}=0.033$; Fig. 3B). p15, a target gene of the TGF- $\beta /$ Smad pathway, exhibited significantly increased expression in KLF10-KO mice compared with WT mice at $72 \mathrm{~h}$ post-PH $(\mathrm{P}=0.049$; Fig. 3C). By contrast, cMyc and cyclin D1 genes, which are suppressed following activation of the TGF- $\beta /$ Smad pathway, exhibited significantly greater expression in WT mice compared with KLF10-KO mice from 48 h post-PH (cMyc, 48 h, $\mathrm{P}=0.042 ; 72$ h, $\mathrm{P}=0.011$ : cyclin $\mathrm{D} 1$, 48 h, $\mathrm{P}=0.036 ; 72$ h, $\mathrm{P}=0.047$; Fig. 3D and E). No significant differences were observed in the mRNA levels of other genes (data not shown).

Expression of proteins involved in the TGF- $\beta /$ Smad pathway. The expression of proteins targeted by the TGF- $\beta$ pathway (Smad2, Smad3, Smad4, Smad7, p15, p21, p27, TGF- $\beta 1$, TGF- $\beta$ R1, TGF- $\beta$ R2 and cyclin D1) was then investigated. In RT-qPCR analysis, significant differences were mostly observed at 48 and $72 \mathrm{~h}$ post-PH. The tissue lysates from these time points were analyzed by western blotting. The results for Smad2, Smad3 and cyclin D1 were similar to those obtained by RT-qPCR. Smad2 expression was significantly deacreased in KLF10-KO mice at 48 and $72 \mathrm{~h}$ post-PH compared with that in WT mice $(\mathrm{P}=0.049$ and $\mathrm{P}=0.043$, respectively; Fig. 4A). Protein levels of Smad3 and p15 were increased in the KLF10-KO mice compared with those in the WT mice, and significant differences were observed at $72 \mathrm{~h}$ post- $\mathrm{PH}(\mathrm{P}=0.019$ and $\mathrm{P}=0.040$, respectively; Fig. 4B and $\mathrm{C}$ ). The cyclin $\mathrm{D} 1$ level was significantly lower at 48 and $72 \mathrm{~h}$ post-PH in the KLF10-KO mice 


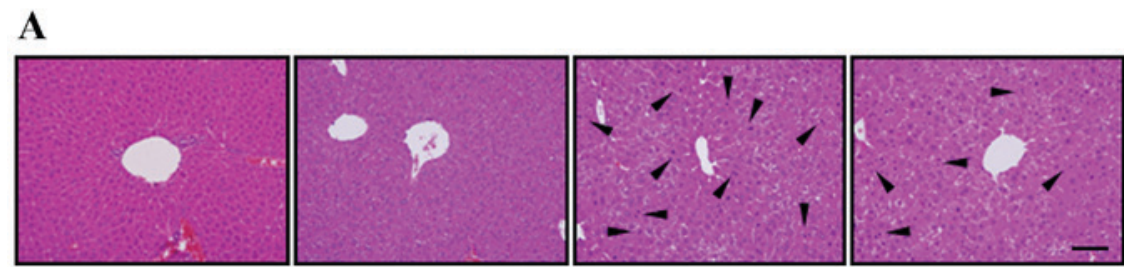

B

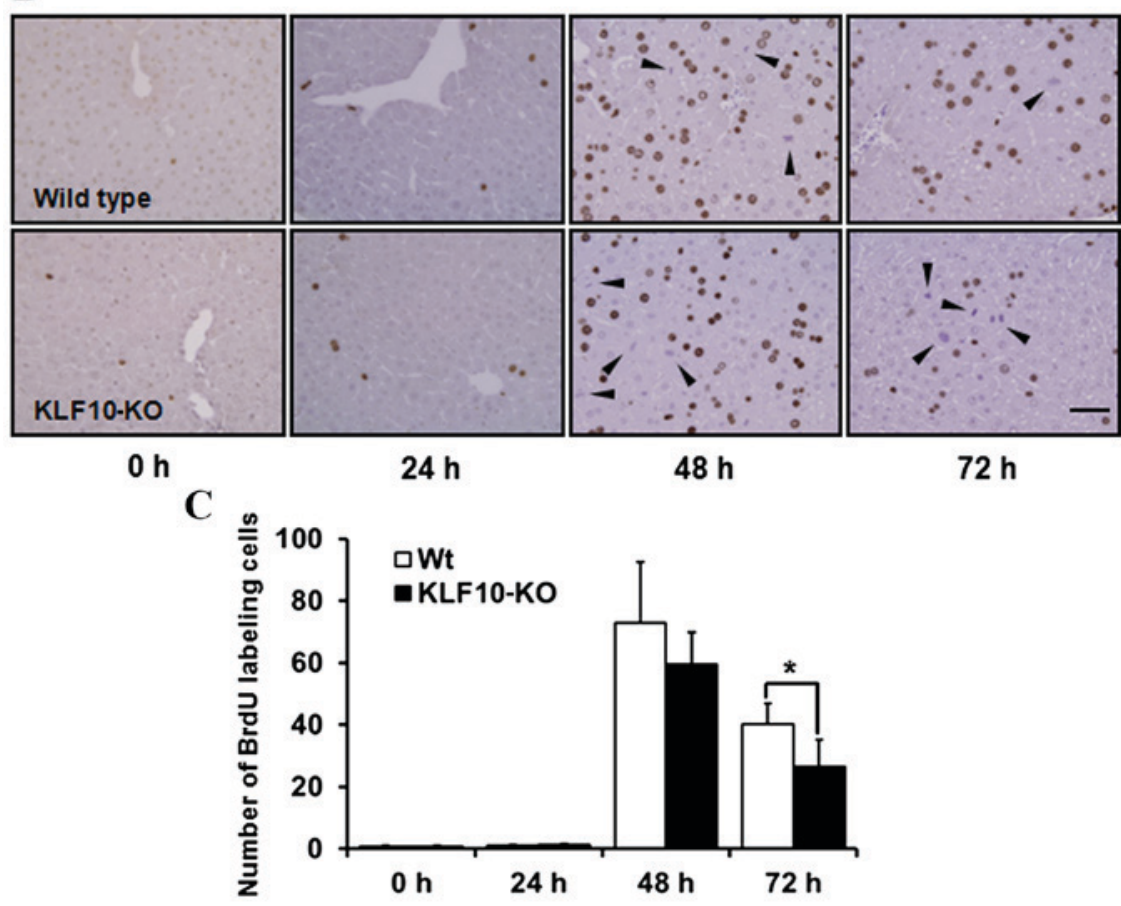

Figure 2. Histopathological findings and BrdU stains of the liver tissue following partial hepatectomy. (A) Hematoxylin and eosin stains of $0,24,48$ and $72 \mathrm{~h}$ post-PH. From $48 \mathrm{~h}$ post-PH, pale cytoplasm and hypertropy of hepatocytes was observed. Mitotic figures, mainly in the centrilobular area, were the most frequent at $48 \mathrm{~h}$ post-PH. Arrowheads indicate mitotic figures. Bar, $100 \mu \mathrm{m}$. (B) Immunohistochemical staining of BrdU in the liver tissue after PH. Nuclei of proliferating cells were numerous in WT mice from $48 \mathrm{~h}$ post-PH. Mitotic cells were coarsely stained (arrowheads). Bar, $50 \mu \mathrm{m}$. (C) BrdU indices of liver tissue. BrdU-positive nuclei were counted in an area of $0.1 \mathrm{~mm}^{2}$ and the number of mitotic cells was added. KLF10-KO mice showed lower indices compared with WT mice from $48 \mathrm{~h}$ post- $\mathrm{PH}$, and there was a significant difference at $72 \mathrm{~h}$ after $\mathrm{PH}$. "P<0.05 by unpaired Student's t-test. PH, partial hepatectomy; KLF10, krüppel-like factor 10; KO, knockout; WT, wild-type; BrdU, 5-bromo-2-deoxy-uridine.

compared with that in the WT mice $(\mathrm{P}=0.045$ and $\mathrm{P}=0.006$, respectively; Fig. 4D). Additionally, the level of TGF- $\beta 1$, which is positively regulated by the TGF- $\beta /$ Smad pathway (14), was increased in the KLF10-KO mice compared with that in the WT mice, and significant differences were observed at $72 \mathrm{~h}$ post-PH ( $\mathrm{P}=0.012$; Fig. 4E). Expression of TGF- $\beta$ R1, which is involved in the early steps of the TGF- $\beta$ pathway (14), was similar to that of TGF- $\beta 1$ (Fig. 4F). Other proteins did not show any significant differences (data not shown).

\section{Discussion}

Numerous factors may alter liver mass, and a number of signaling pathways are involved in liver regeneration $(1,4,7)$. TGF- $\beta$ is known to suppress cellular proliferation, including hepatic regeneration $(8,9)$. KLF10, one of the target genes of TGF- $\beta$, enhances TGF- $\beta$-induced anti-proliferative effects in certain cell types by inducing the expression of several genes involved in TGF- $\beta$ signaling, including Smads, p15, p21 and TGF- $\beta 1$ (24-26). However, the exact function of KLF10 under various pathophysiological conditions remains unclear, and to the best of our knowledge, its role in liver regeneration has never been examined.

In the present study, the role of KLF10 in liver regeneration following tissue loss was investigated using KLF10-KO mice. The liver/body weight ratio of the KLF10-KO mice was lower than that of the WT mice at all examined time points, and a significant difference was observed at $72 \mathrm{~h}$ post-PH (Fig. 1). KLF10-KO mice also exhibited lower BrdU indices compared with WT mice from $48 \mathrm{~h}$ post-PH, and a significant difference was observed at $72 \mathrm{~h}$ post-PH (Fig. 2C). Thus, cellular proliferation appeared to be suppressed in KLF10-KO mice following $\mathrm{PH}$ (under conditions that supported regeneration in WT mice). To determine the causes for the decreased cellular proliferation in KLF10-KO mice, the expression of genes involved in the TGF- $\beta /$ Smad pathway was examined by RT-qPCR. Under normal conditions and immediately following $\mathrm{PH}$, no difference was observed in the expression of the examined genes. However, the mRNA levels of Smad2 decreased significantly in KLF10-KO mice from $24 \mathrm{~h}$ post-PH (Fig. 3A). KLF10 has been reported to enhance Smad2 expression (10), and this decrease may be a cause for KLF10 gene ablation in the 

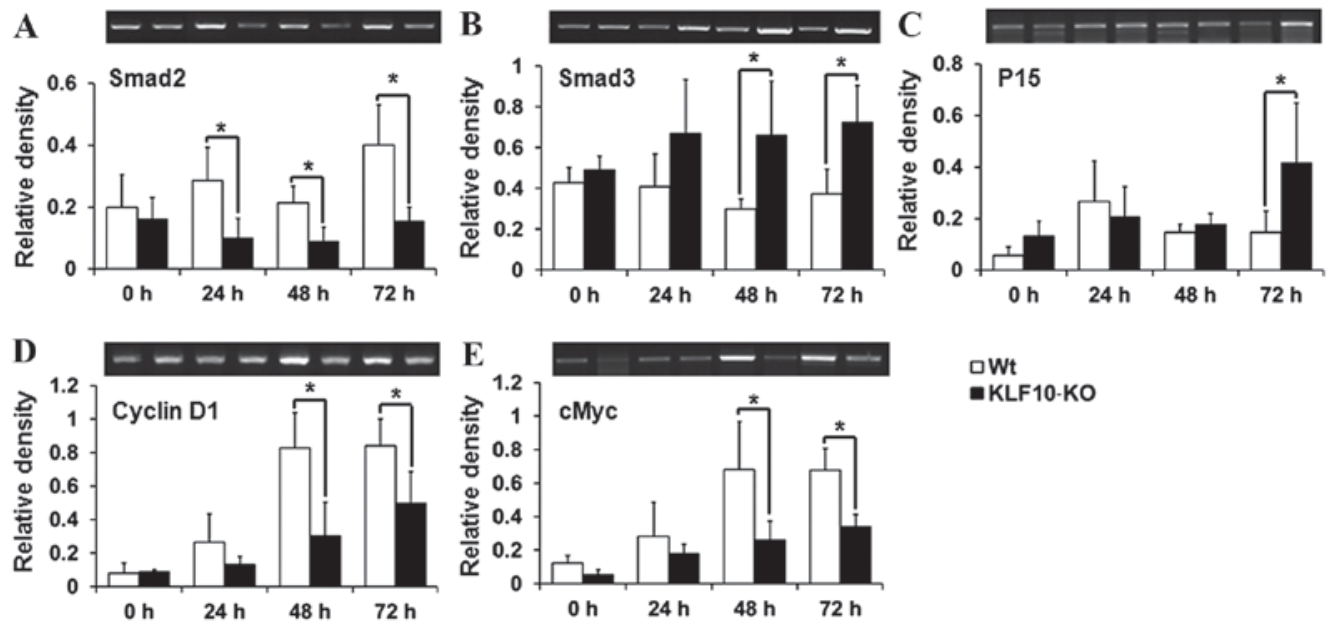

QWt
KLF10-KO

Figure 3. Analysis of the mRNA levels of the genes (Smad2, Smad3, p15, cyclin D1 and cMyc) involved in the transforming growth factor- $\beta$ /Smad pathway. (A) Smad2 was downregulated, while (B) Smad3 and (C) p15 were upregulated in the KLF10-KO mice (D) Cyclin D1 and (E) cMyc were also downregulated. Band intensities were quantified and normalized to the $\beta$-actin level. * $\mathrm{P}<0.05$ by unpaired Student's t-test. KLF10, krüppel-like factor 10 ; KO, knockout; WT, wild-type.
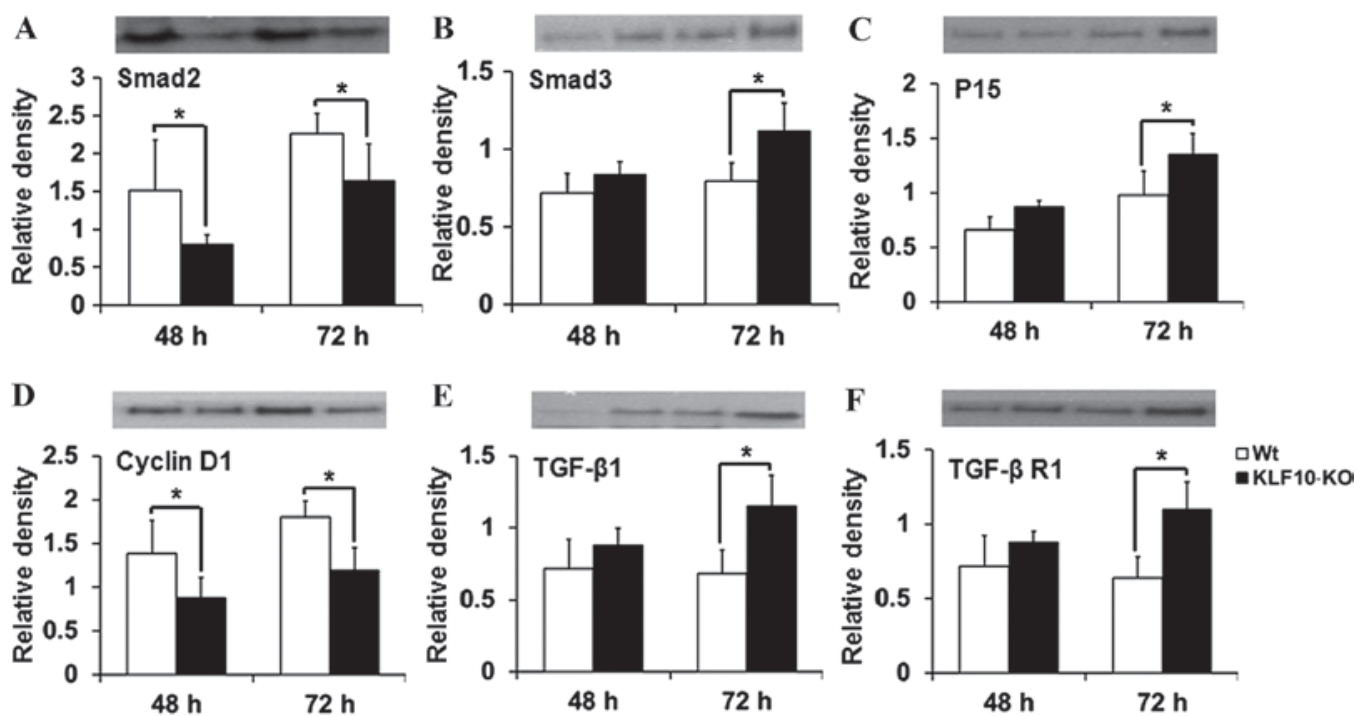

Figure 4. Analysis of the levels of the proteins (Smad2, Smad3, p15, cyclin D1, TGF- $\beta 1$ and TGF- $\beta$ R1) involved in the TGF- $\beta /$ Smad pathway. Considering reverse transcription-quantitative polymerase chain reaction results, liver tissues at 48 and $72 \mathrm{~h}$ post-partial hepatectomy were examined. (A) Smad2 levels were lower, while (B) Smad3 and (C) p15 levels were higher in the KLF10-KO mice compared with those in the WT mice. (D) Cyclin D1 levels were lower, while (E) TGF- $\beta 1$ and (F) TGF- $\beta$ R1 levels were higher in the KLF10 KO mice compared with those in the WT mice. Band intensities were quantified and normalized to the $\beta$-actin level. "P $<0.05$ by unpaired Student's t-test. TGF- $\beta 1$, transforming growth factor- $\beta 1$; TGF- $\beta$ R1, transforming growth factor- $\beta$ receptor 1; KLF10, krüppel-like factor 10; KO, knockout; WT, wild-type.

KO mice. Unlike that of Smad2, the expression of Smad3 (a co-Smad) increased from $48 \mathrm{~h}$ post-PH in the KLF10-KO mice (Fig. 3B). The expression of p15, whose transcription is induced by the activated Smads complex, also increased from $48 \mathrm{~h}$ post-PH in the KLF10-KO mice (Fig. 3C). Consequently, the cMyc and cyclin D1 genes, which are downregulated by p15, showed significantly lower expression in the KLF10-KO mice compared with the WT mice from $48 \mathrm{~h}$ post-PH (Fig. 3D and E). Western blot analysis results for Smad2, Smad3, p15 and cyclin D1 were similar to those of RT-qPCR. In addition, the levels of TGF- $\beta 1$ and TGF- $\beta$ R1, which are involved in the early phases of the TGF- $\beta$ pathway, were significantly increased at $72 \mathrm{~h}$ post-PH in the KLF10-KO mice compared with the WT mice (Fig. 4).
These results indicated that the knockout of KLF10 led to repression of the transcription and function of the proliferation-associated genes cMyc and cyclin D1 from $48 \mathrm{~h}$ post-PH, thereby inhibiting liver regeneration. This repression may be induced by the activation of the TGF- $\beta /$ Smad pathway. Expression of TGF- $\beta$ R1, TGF- $\beta 1$, Smad 3 and p15, one of the genes positively regulated by the TGF- $\beta$ pathway, was increased in the KLF10-KO mice. Thus, during liver regeneration following PH, lack of KLF10 suppresses the proliferation of hepatocytes by activation of the TGF- $\beta /$ Smad pathway, and the suppression of hepatocellular proliferation can be observed in a delayed manner at $72 \mathrm{~h}$ post-PH.

Cellular responses to TGF- $\beta$ depend on the cell types, the microenvironment and the tissue type $(9,27)$. KLF10, 
an early target of TGF- $\beta$, has been reported to exert effects similar to those of TGF- $\beta$. However, the specific functions of KLF10 under various physiological conditions are not well characterized $(18,26,28,29)$. Our previous study reported the tumor-suppressor effects against chemically-induced liver tumorigenesis in KLF10-KO mice (12). Similarly, the anti-proliferative effects on hepatocytes after $\mathrm{PH}$ were shown in the present study. These results show that although there were no phenotypical changes in the liver of KLF10-KO mice under normal physiological conditions, the TGF- $\beta /$ Smad pathway exhibited enhanced activation and cellular proliferation was decreased under conditions that promote cellular proliferative changes in the liver, including $\mathrm{PH}$ and chemically-induced tumorigenesis.

The aforementioned results may be due to KLF10 transcription being induced by factors other than TGF- $\beta$, including estrogen and epidermal growth factor $(8,14,30,31)$. Furthermore, certain intracellular proteins, including KLF11, were recently reported to exhibit effects similar to those of KLF10 (26,32). Considering these findings, certain compensatory mechanisms for KLF10 ablation may exist in non-Smad TGF- $\beta$ pathways or KLF10 signaling under proliferative conditions. The detailed molecular mechanism underlying this compensatory mechanism warrants additional study.

In conclusion, the hepatocytes of KLF10-KO mice exhibited less proliferation compared with those of WT mice following $\mathrm{PH}$ in the present study. This decrease was caused by reinforcement of TGF- $\beta /$ Smad signaling. TGF- $\beta 1$, TGF- $\beta$ R1 and Smad3 were upregulated, and this induced increased expression of $\mathrm{p} 15$. These factors led to repressed expression of the proliferation-associated genes, cMyc and cyclin D1. The present findings demonstrated that knockout of KLF10 suppressed cellular proliferation through reinforcement of the TGF- $\beta /$ Smad pathway in the presence of external stimuli that could induce hepatocyte proliferation, including tumorigenesis and regeneration, following tissue loss (12).

\section{Acknowledgements}

The present study was supported by the Konkuk University in 2013.

\section{References}

1. Fausto N, Campbell JS and Riehle KJ: Liver regeneration. J Hepatol 57: 692-694, 2012.

2. Fausto N: Liver regeneration. J Hepatol 32 (1 Suppl): S19-S31, 2000.

3. Michalopoulos GK: Liver regeneration. J Cell Physiol 213: 286-300, 2007

4. Michalopoulos GK: Liver regeneration after partial hepatectomy: Critical analysis of mechanistic dilemmas. Am J Pathol 176: 2-13, 2010.

5. Maluccio M and Covey A: Recent progress in understanding, diagnosing and treating hepatocellular carcinoma. CA Cancer J Clin 62: 394-399, 2012.

6. Mathers CD, Boerma T and Ma Fat D: Global and regional causes of death. Br Med Bull 92: 7-32, 2009.

7. Taub R: Liver regeneration: From myth to mechanism. Nat Rev Mol Cell Biol 5: 836-847, 2004.

8. Derynck R, Akhurst RJ and Balmain A: TGF-beta signaling in tumor suppression and cancer progression. Nat Genet 29: $117-129,2001$.
9. Itoh S and ten Dijke P: Negative regulation of TGFbeta receptor/Smad signal transduction. Curr Opin Cell Biol 19: 176-184, 2007.

10. Subramaniam M, Hawse JR, Rajamannan NM, Ingle JN and Spelsberg TC: Functional role of KLF10 in multiple disease processes. Biofactors 36: 8-18, 2010.

11. Ellenrieder V: TGFbeta-regulated gene expression by smads and Sp1/KLF-like transcription factors in cancer. Anticancer Res 28: 1531-1539, 2008.

12. Heo SH, Jeong ES, Lee KS, Seo JH, Lee WK and Choi YK: Krüppel-like factor 10 null mice exhibit lower tumor incidence and suppressed cellular proliferation activity following chemically induced liver tumorigenesis. Oncol Rep 33: 2037-2044, 2015.

13. Inman GJ: Switching TGF $\beta$ from a tumor suppressor to a tumor promoter. Curr Opin Genet Dev 21: 93-99, 2011.

14. Massagué J: TGFbeta in Cancer. Cell 134: 215-230, 2008.

15. Kaminska B, Wesolowska A and Danilkiewicz M: TGF beta signalling and its role in tumour pathogenesis. Acta Biochim Pol 52: 329-337, 2005.

16. Kang JS, Liu C and Derynck R: New regulatory mechanisms of TGF-beta receptor function. Trends Cell Biol 19: 385-394, 2009.

17. Johnsen SA, Subramaniam M, Monroe DG, Janknecht R and Spelsberg TC: Modulation of transforming growth factor beta (TGF-beta)/Smad transcriptional responses through targeted degradation of TGF-beta-inducible early gene-1 by human seven in absentia homologue. J Biol Chem 277: 30754-30759, 2002.

18. Subramaniam M, Hawse JR, Johnsen SA and Spelsberg TC: Role of TIEG1 in biological processes and disease states. J Cell Biochem 102: 539-548, 2007.

19. Martins PN, Theruvath TP and Neuhaus P: Rodent models of partial hepatectomies. Liver Int 28: 3-11, 2008.

20. Mitchell C and Willenbring H: A reproducible and well-tolerated method for $2 / 3$ partial hepatectomy in mice. Nat Protoc 3: 1167-201170,2008.

21 . Breitkopf $\mathrm{K}$, Weng $\mathrm{H}$ and Dooley S: TGF- $\beta /$ Smad-signaling in liver cells: Target genes and inhibitors of two parallel pathways. Signal Transduction 6: 329-337, 2006.

22. Song KD, Kim DJ, Lee JE, Yun CH and Lee WK: KLF10, transforming growth factor- $\beta$-inducible early gene 1 , acts as a tumor suppressor. Biochem Biophys Res Commun 419: 388-394, 2012.

23. Shiao YH: A new reverse transcription-polymerase chain reaction method for accurate quantification. BMC Biotechnol 3: 22, 2003.

24. Buenemann CL, Willy C, Buchmann A, Schmiechen A and Schwarz M: Transforming growth factor-beta1-induced Smad signaling, cell-cycle arrest and apoptosis in hepatoma cells. Carcinogenesis 22: 447-452, 2001.

25. Dooley S, Weng H and Mertens PR: Hypotheses on the role of transforming growth factor-beta in the onset and progression of hepatocellular carcinoma. Dig Dis 27: 93-101, 2009.

26. Spittau B and Krieglstein K: Klf10 and Klf11 as mediators of TGF-beta superfamily signaling. Cell Tissue Res 347: 65-72, 2012.

27. Heldin $\mathrm{CH}$ and Miyazono K: Transforming growth factor-beta. An interesting candidate for clinical use. Lakartidningen 92: 1569-1572, 1995 (In Swedish).

28. Bensamoun SF, Hawse JR, Subramaniam M, Ilharreborde B, Bassillais A, Benhamou CL, Fraser DG, Oursler MJ, Amadio PC, An KN and Spelsberg TC: TGF-beta inducible early gene-1 knockout mice display defects in bone strength and microarchitecture. Bone 39: 1244-1251, 2006.

29. Bos JM, Subramaniam M, Hawse JR, Christiaans I, Rajamannan NM, Maleszewski JJ, Edwards WD, Wilde AA, Spelsberg TC and Ackerman MJ: TGF $\beta$-inducible early gene-1 (TIEG1) mutations in hypertrophic cardiomyopathy. J Cell Biochem 113: 1896-1903, 2012.

30. Moustakas A and Heldin CH: Non-Smad TGF-beta signals. J Cell Sci 118: 3573-3584, 2005.

31. Tsubone T, Moran SL, Subramaniam M, Amadio PC, Spelsberg TC and An KN: Effect of TGF-beta inducible early gene deficiency on flexor tendon healing. J Orthop Res 24: 569-575, 2006.

32. Gohla G, Krieglstein K and Spittau B: Tieg3/K1f11 induces apoptosis in OLI-neu cells and enhances the TGF-beta signaling pathway by transcriptional repression of Smad7. J Cell Biochem 104: 850-861, 2008. 